\title{
Newton trajectories in the curvilinear metric of internal coordinates
}

\author{
Wolfgang Quapp \\ Mathematical Institute, University of Leipzig, Augustus-Platz, D-04109 Leipzig, Germany \\ E-mail: quapp@rz.uni-leipzig.de
}

Received 19 February 2004; revised 5 July 2004

\begin{abstract}
The reaction path is an important concept of theoretical chemistry. We discuss the definition with the help of the following of the reduced gradient (RGF) [see Quapp et al., Theoret. Chem. Acc. 100 (1998) 285], also named the Newton trajectory. All the important features of the potential energy surface are definable independently of the coordinate system. We demonstrate it for the Newton trajectory. We design a numerical scheme for the RGF method including the intrinsic curvilinear metric of internal coordinates. For the path following we extend the previous method to the use of a generalized singular value decomposition (SVD). An example of the $\mathrm{HCN}$ isomerization pathway is discussed.
\end{abstract}

KEY WORDS: Potential energy surface, reaction path following, projected gradient, valley-ridge inflection point, singular value decomposition

AMS subject classification: Primary: 58C15, 15A18

Secondary: 53A04, 53B21, 53Z04, 58-04

\section{Introduction}

The concept of the minimum energy path (MEP) or reaction path (RP) of an adiabatic potential energy surface (PES) is the usual approach to the theoretical kinetics of larger chemical systems [1-6]. It is roughly defined as a line in coordinate space, which connects two minima by passing the saddle point (SP) (the transition structure) of a PES. The energy of the SP is assumed to be the highest value tracing along the RP. It is the minimal energy a reaction needs to take place. Reaction theories are based either implicitly (transition state theory [1]), or explicitly (variational transition state theory[5]) on the knowledge of the RP. These theories require only local information about the PES along the RP. They circumvent the dimension problem for medium-sized or large molecules: it is impossible to fully calculate their PES. Any parameterization $s$ of the RP $\mathbf{x}(s)=\left(x^{1}(s), \ldots, x^{n}(s)\right)^{T}$ is called reaction coordinate. It is an uncertainty of the RP definition, how a reaction path ascends to the SP. We use here the distinguished or driven coordinate method [7] in the modern form of RGF [8-13]. 
We remark that the search for an appropriate MEP is not necessary equivalent to the finding of the steepest descent (SD) pathway from the SP.

Reaction paths are a widely used concept in theoretical chemistry. Properties of such paths should be independent of the coordinate system employed [3, $6]$. If this were not the case we would obtain different dynamic and thermodynamic properties from different coordinates. This seems like a trivial point, and we are sorry to belabor it. We will develop the corresponding formulas for RGF pathways which are called Newton trajectories. Some of the methods are implemented as independent modular programs. The programs can be obtained on request or retrieved [14].

\section{Definition of RGF curves}

The starting point is a geometrically defined pathway which may serve as a reaction path. Geometrically defined means that only properties of the PES are taken into account like in the SD, however, no dynamical behavior of the molecule is observed. It was proposed to choose a distinguished coordinate along the valley of the minimum, to go a step in this direction, and to perform an energy optimization of the residual coordinates. However, the methodology of the distinguished coordinate method was criticized by some workers: Müller [15], Müller and Brown [16], Williams and Maggiora [7], and Cioslowski et al. [17]. They found examples, where the distinguished coordinate method fails: it cannot follow the path over a turning point. Recently, the method was transformed into a new mathematical form to $\operatorname{RGF}[8,9]$. The concept is that any selected gradient direction is fixed

$$
\mathbf{g}(\mathbf{x}) /|\mathbf{g}(\mathbf{x})|=\mathbf{r},
$$

where $\mathbf{r}$ is the unit vector of the search direction. The search direction may correspond to an assumed start direction of a chemical reaction. For example, it may be the direction between the two minima of reactant and product. The property (1) is also realizable by a projection of the gradient onto the $(n-1)$-dimensional subspace which is orthogonal to the one-dimensional subspace spanned by the search direction $\mathbf{r}$. A curve belongs to the search direction $\mathbf{r}$, if the gradient of the PES always remains parallel to the direction of $\mathbf{r}$ at every point along the curve $\mathbf{x}(s)$ with a suitable parameter $s$

$$
{ }^{(n-1)} \mathbf{P}^{\mathbf{r}} \mathbf{g}(\mathbf{x}(s))=\mathbf{0},
$$

where ${ }^{(n-1)} \mathbf{P}^{\mathbf{r}}$ projects out the search direction $\mathbf{r}$. This means ${ }^{(n-1)} \mathbf{P}^{\mathbf{r}} \mathbf{r}=\mathbf{0}$. The projector ${ }^{(n-1)} \mathbf{P}^{\mathbf{r}}$ in equation (2) "reduces" the gradient. It is to be constructed by $(n-1)$ row vectors being orthogonal to the search direction $\mathbf{r}$. They can be obtained by a modified Gram-Schmidt orthogonalization [18]. Based on the explicit definition, we can follow curve (2) along its tangential vector. This is the 
RGF method (the reduced gradient following) using the derivative of equation (2) to obtain the tangent $\mathbf{x}^{\prime}$ to the curve

$$
\mathbf{0}=\frac{\mathrm{d}}{\mathrm{d} s}\left[\mathbf{P}^{\mathrm{r}} \mathbf{g}(\mathbf{x}(s))\right]=\mathbf{P}^{\mathbf{r}} \frac{\mathrm{d} \mathbf{g}(\mathbf{x}(s))}{\mathrm{d} s}=\mathbf{P}^{\mathbf{r}} \mathbf{H}(\mathbf{x}(s)) \mathbf{x}^{\prime}(s) .
$$

The matrix $\mathbf{H}$ is the Hessian. The algorithm has already been realized by the predictor-corrector method $[9,19]$ using ${ }^{(n-1)} \mathbf{P}^{\mathbf{r}}$. The RGF is a simple but effective procedure in order to determine all types of stationary points [8]. In most of the cases, each RGF curve passes each stationary point [20]. RGF curves are defined by a constant gradient vector, and a full family of them connects the extrema. Nevertheless, some of the curves may follow a reaction valley in favorable cases. The possibility of the MEP calculation then depends on a clever definition of the search direction [21].

If working in a curvilinear coordinate space the projection into an $(n-1)$ dimensional subspace in equation (2) may obscure the structure of the metric. If the zero in equation (2) is exactly fulfilled, a curve point is given. However, if equation (2) is not fulfilled then the $(n-1)$ dimensional vector in equation (2) is to be measured in an unknown metric. One remedy is the exact use of the internal metric, see section 6 . Another way is to define a new $\mathbf{P}^{\mathbf{r}}$ by the dyadic product of the unit vector $\mathbf{r}$ with $\mathbf{r}^{T}$

$$
{ }^{n} \mathbf{P}^{\mathbf{r}}=\mathbf{I}_{n}-\mathbf{r r}{ }^{T},
$$

where $\mathbf{I}_{n}$ is the unit matrix. Again, we pose

$$
{ }^{n} \mathbf{P}^{\mathbf{r}} \mathbf{g}(\mathbf{x}(s))=\mathbf{0} .
$$

This ${ }^{n} \mathbf{P}^{\mathbf{r}}$ is an $n \times n$ matrix of rank $n-1$, because $\mathbf{r}$ is a column vector, $\mathbf{r}^{T}$ is a row vector, and their dyadic product is a matrix. For projector (4) we can measure the zero of equation (5) directly in the $n$-dimensional curvilinear metric. This version of ${ }^{n} \mathbf{P}^{\mathbf{r}}$ is used in section 7 .

The turning point (TP) case $[7,16,20]$ divides RGF curves into those which can serve as loose RPs, and others: if the RGF curve does not contain a TP at the pathway from minimum up to the SP, it may be used as an RP model. However, if a TP emerges, it will usually have an energy higher than the energy of the respective SP and, hence, this path does not meet the meaning of an RP.

\section{Description of the first RGF algorithm}

In order to get the system of equations for RGF, we have to define the projector ${ }^{(n-1)} \mathbf{P}^{\mathbf{r}}$. In procedure mrgf2 [14], we calculate $(n-1)$ orthonormal direction vectors being also orthogonal to the selected search direction $\mathbf{r}$ by using the modified Gram-Schmidt algorithm [18]. (The metric of internal coordinates may be included into the orthogonality relation of the Gram-Schmidt procedure.) 
Then, the projector ${ }^{(n-1)} \mathbf{P}^{\mathbf{r}}$ is the matrix of these $(n-1)$ rows. Its action in equation (2) is measured in an Euclidean, $(n-1)$-dimensional norm. The equation (3) for RGF becomes ${ }^{(n-1)} \mathbf{P}^{\mathbf{r}} \mathbf{H} \mathbf{t}=\mathbf{0}$, a linear equation for the tangent vector $\mathbf{t}$. It makes up the predictor step. The system is solved by QR decomposition [19]. The reduced Hessian ${ }^{(n-1)} \mathbf{P}^{\mathbf{r}} \mathbf{H}$, an $(n-1) \times n$ matrix, is augmented by the tangent vector to an $(n \times n)$ matrix which is the so-called $\mathbf{K}$ matrix [19]. The corrector step is applied if the Euclidean norm of the reduced gradient ${ }^{(n-1)} \mathbf{P}^{\mathbf{r}} \mathbf{g}_{I}$ is greater than a threshold $\epsilon$ which is given as a parameter. The subsequent Newton-Raphson step of the corrector orthogonally to the tangent is realized by solving a linear equation where the $\mathbf{K}$ matrix forms the left hand side, and the right-hand side is given by the reduced gradient augmented by zero in the $n$-th row. Either the predictor step, or the corrector step is added to the current $\mathbf{z}$ matrix values of the internal coordinates, and the next loop of the algorithm is started. (A combination of predictor and corrector gives even better results [22].) RGF is now tested to be an effective tool in determining the next SP [11-13,20,23-25], on a PES if starting at a minimum. As long as it was used to find stationary points, the coordinate system did not matter so much; however, RGF curves can also be defined independently of coordinates, see below.

\section{Branin's method to calculate symmetric valley-ridge inflection points}

The gradient directions of the PES are uniquely determined. Curves calculated by RGF to different directions $\mathbf{r}$ cross if and only if the gradient vanishes at the crosspoint, i.e., the crosspoint has to be a stationary point. However, different branches of the solution of the same reduced gradient curve with respect to $\mathbf{r}$ may also cross each other. These points are characterized as the branching points (BP) of the reduced gradient curve. On the other hand, the BP of a reaction path is an interesting issue in theoretical chemistry (see [26] and references therein). The branching of an RGF curve is connected with the emergence of special points of the PES, the valley-ridge inflection (VRI) points of the surface $[9,27,28]$. The traditional definition is that a VRI point is that point in the configuration space where, orthogonally to the gradient, at least one main curvature of the PES becomes zero. Usually, VRI points represent non-stationary points of the PES. VRI points in the narrow sense of this definition are defined independently of an RP definition. However, whenever a reduced gradient curve reaches a VRI point, the curve branches, and at every VRI point of the PES the solution of a corresponding reduced gradient curve branches [9]. However, not every RGF curve has a BP. (The path following of those RGF curves which have a BP allows to find VRI points.)

The RGF approach shows an analogy to the mathematical theory of Branin [29], the global Newton method [30,31]. It utilizes the adjoint matrix $\mathbf{A}$ of the Hessian matrix $\mathbf{H}$. This is defined as $\left((-1)^{i+j} m_{i j}\right)^{T}$ where $m_{i j}$ is the minor 
of $\mathbf{H}$ obtained by deletion of the $i$ th row and the $j$ th column from $\mathbf{H}$, and taking the determinant. The adjoint matrix satisfies the relation

$$
\mathbf{H A}=\operatorname{Det}(\mathbf{H}) \mathbf{I}_{n},
$$

where $\operatorname{Det}(\mathbf{H})$ is the determinant of $\mathbf{H}$, and $\mathbf{I}_{n}$ is the unit matrix. The adjoint matrix $\mathbf{A}$ is used to define an autonomous system of differential equations for the curve $\mathbf{x}(s)$, where $s$ is a curve parameter:

$$
\frac{\mathrm{d} \mathbf{x}}{\mathrm{d} s}= \pm \mathbf{A}(\mathbf{x}) \mathbf{g}(\mathbf{x}) .
$$

The "+" option is used for searching stationary points with an odd index (SPs with an odd number of negative eigenvalues of the Hessian), where the "-" option searches for stationary points with an even index. Because solutions of a differential equation are named trajectories, the solutions of (7) are named Newton trajectories. They are the same curves like RGF curves using the same gradient [9].

The Branin method is a fine tool to find symmetric VRI points as exactly as we need them. For the calculation one chooses the steplength parameter, $l$, by trial and error and discretizes Branin's differential equation (7) to

$$
\mathbf{x}_{\mathbf{m}+\mathbf{1}}=\mathbf{x}_{\mathbf{m}} \pm l \mathbf{A}_{\mathbf{m}} \mathbf{g}_{\mathbf{m}},
$$

where $m$ is the step number $[9,10,25,29,32] . \mathbf{A}_{\mathbf{m}}$ is the adjoint matrix of the Hessian and $\mathbf{g}_{\mathbf{m}}$ is the gradient at point $\mathbf{x}_{\mathbf{m}}$. Two-dimensional VRI manifolds were detected lying in the 3-D subspace of symmetry coordinates of $\mathrm{H}_{2} \mathrm{CO}$ [32].

\section{Invariance of coordinate system}

It is evident that the invariance problem of the MEP, which has mathematically been solved a long time ago [3] and report [6], penetrates the discussions in this field again and again [33-36]. In a reaction, each atom describes its own pathway in the 3-D Cartesian space, and the total movement of $N$ atoms of the molecular system defines the migration of a point in the configuration space $\mathrm{R}^{3 N}$, say by coordinates $y^{i}, i=1, \ldots, 3 N$. One needs coordinate invariance for any mass-weighted internal coordinates $x^{k}=x^{k}\left(y^{i}\right), k=1, \ldots, n$, with $n=3 N-6$. They are given by a $\mathbf{z}$ matrix. The corresponding $\mathbf{B}$ matrix [37] is

$$
\mathbf{B}=\left(\frac{\partial x^{k}}{\partial y^{i}}\right), \quad k=1, \ldots, n, \quad i=1, \ldots, n+6
$$

with $n$ rows and $(n+6)$ columns. The PES $E\left(x^{1}, \ldots, x^{n}\right)$ is a scalar depending on the coordinates $x^{k}$ of the molecule. The coordinates are assumed as the contravariant vector $\mathbf{x}=\left(x^{1}, \ldots, x^{n}\right)^{T}$. Each derivation of $E(\mathbf{x})$ to $x^{k}$, with 
$k=1, \ldots, n$, yields a component of a vector, termed the gradient vector of $E$. $\partial E / \partial x^{k}$ takes the $k$-th place in the gradient $\mathbf{g}_{I}=\left(g_{1}, \ldots, g_{n}\right)^{T}$ which is a covariant vector $[6,38]$. The $\mathbf{B}$ matrix serves as a linear approximation of the transformation

$$
\mathbf{g}_{C}=\mathbf{B}^{T} \mathbf{g}_{I}
$$

for the change of the gradients in Cartesian or internal coordinates, and

$$
\mathbf{H}_{C}=\mathbf{B}^{T} \mathbf{H}_{I} \mathbf{B}+\Gamma
$$

for the change of the Hessians. The $\Gamma$ term comes out of the chain rule to

$$
\Gamma_{i j}=\sum_{k}\left(\mathbf{g}_{I}\right)_{k} \frac{\partial^{2} x^{k}}{\partial y^{i} \partial y^{j}} .
$$

Explicit derivatives of the $\mathbf{B}$ matrix to the Cartesian coordinates are given [39, 40]. The transformation of $\mathbf{g}_{C}$ to the gradient $\mathbf{g}_{I}$ inverse to (10) can go on first by a multiplication from the left-hand side with $\mathbf{B}$ and second with the use of the inverse of the regular ( $\mathbf{B} \mathbf{B}^{T}$ ) matrix:

$$
\left.(\mathbf{B ~ B})^{T}\right)^{-1} \mathbf{B g}_{C}=\mathbf{g}_{I} .
$$

The transformation matrix on the left-hand side is the "left inverse" of $\mathbf{B}^{T}$. It is called the pseudoinverse matrix $\mathbf{B}^{+}$, cf. [41] for its use in spectroscopy. It is also an $n \times(n+6)$ matrix. The contravariant metric tensor $\mathbf{G}^{-1}=\left(g^{i j}\right)$ is calculated point by point along the pathway of an MEP taking $\mathbf{B} \mathbf{B}^{T}$ where the usual metric tensor $\mathbf{G}=\left(g_{i j}\right)$ forms its inverse matrix. The pseudoinverse is $\mathbf{B}^{+}=\mathbf{G} \mathbf{B}$. The gradient $\mathbf{g}_{C}$ and the Hessian matrix $\mathbf{H}_{C}$ are calculated in Cartesian coordinates by most quantum chemical programs. However, at any point they are transformed to their internal version by $\mathbf{B}^{+} \mathbf{g}_{C}=\mathbf{g}_{I}$ and $\mathbf{B}^{+} \mathbf{H}_{C}\left(\mathbf{B}^{+}\right)^{T}=\mathbf{H}_{I}$ (where we usually ignore the $\Gamma$ term in (11) for the Hessian, because it is usually small).

\section{The invariance of RGF and of Branin's equation}

In internal curvilinear coordinates we have the covariant version of the gradient in equation (1), and we have also to use a covariant vector for the selected direction $\mathbf{r}$. It is intuitively plain that the projector (2) can be defined by orthogonal vectors in a correct sense of the internal metric. The means is the modified Gram-Schmidt (MGS) method [18] with the internal metric of the corresponding scalar products. Setting $\mathbf{p}_{n}=\mathbf{r}$, we get an orthonormal system of covariant vectors $\left\{\mathbf{p}_{1}, \ldots, \mathbf{p}_{n}\right\}$ with relation

$$
\mathbf{p}_{k}^{T} G^{-1} \mathbf{p}_{l}=\delta_{k l}, \quad k, l=1, \ldots, n .
$$


Starting point for the projector (2) is the row matrix $\mathbf{P}$ of this basis, reduced by the last row, the $\mathbf{r}$ vector. But a next step is necessary; we have to build the contravariant components of the vectors by

$$
\mathbf{P} G^{-1}=:^{(n-1)} \mathbf{P}^{r} .
$$

It is again a matrix of $(n-1)$ rows and $n$ columns. One may name the rows of the new matrix by $\mathbf{p}^{k}$ symbols to hint for the contravariant character. By construction, it is

$$
{ }^{(n-1)} \mathbf{P}^{r} \mathbf{r}=\mathbf{0} .
$$

(The zero vector on the right-hand side is $(n-1)$-dimensional.) We also build $\mathbf{r}^{\text {contra }}$, the contravariant components of $\mathbf{r}=\mathbf{r}_{\text {cov }}$. It is

$$
r^{i}=\sum_{j=1}^{n} \mathrm{~g}^{i j} r_{j} .
$$

It holds

$$
\sum_{j=1} r^{j} r_{j}=\sum_{i, j=1} \mathrm{~g}^{i j} r_{i} r_{j}=1
$$

$\mathbf{r}$ is a unit vector in the curvilinear space. If $\mathbf{a}$ is any vector given by its covariant components like the gradient, we can construct the projection of it by ${ }^{(n-1)} \mathbf{P}^{r}$, as well as by $\mathbf{r}^{\text {contra. }}$. We will get $\mathrm{n}$ numbers:

$$
\mathbf{p}^{k} \mathbf{a}=a^{k}, \quad k=1, \ldots, n-1, \quad \mathbf{p}^{n} \mathbf{a}=\mathbf{r}^{\mathrm{contra}} \mathbf{a}=a^{n} .
$$

Because the $\mathbf{p}^{k}$ are also an orthonormal basis, the numbers $\mathrm{a}^{k}$ are contravariant, as well as covariant components of the vector a. (The definitions of the two components are in accord.) We may represent the covariant a by the system of covariant basis vectors:

$$
\mathbf{a}=\sum_{k}^{n-1} \mathbf{p}_{k} a^{k}+\mathbf{r} a^{n} .
$$

The norm of $\mathbf{a}$ is simply the Cartesian sum

$$
\|\mathbf{a}\|=\left(\sum_{k}^{n}\left(a^{k}\right)^{2}\right)^{1 / 2}
$$

using equation (14), and the norm of the projection of $\mathbf{a}$ orthogonal to $\mathbf{r}$ is this sum up to $(n-1)$ terms. Consequently, the projector is a mixed co- and contravariant tensor. The projector has to be recalculated at new curve points because the metric changes from point to point. Note that the metric usually changes 
slowly along an RP, then the change of the $\mathbf{p}^{k}$ is also slowly. By its construction, the projector ${ }^{(n-1)} \mathbf{P}^{\mathbf{r}}$ may be seen to be an invariant tensor under coordinate transformation. It is applicable to the covariant gradient in equation (2), as well as to the two-fold covariant Hessian in equation (3), but it does not change the character of its argument after application. Then an RGF calculation is invariant under coordinate transformation. The condition equation (2) defines a onedimensional curve of points, $\mathbf{x}(s)$ which are contravariant vectors. The aim of a path following procedure is the calculation of $\mathbf{x}(s)$. The tangent for the predictor step is given by

$$
\mathbf{0}=\frac{\mathrm{d}}{\mathrm{d} s}\left[\mathbf{P}^{\mathbf{r}} \mathbf{g}(\mathbf{x}(s))\right]=\left[\frac{\mathrm{d} \mathbf{P}^{\mathbf{r}}}{\mathrm{d} s} \mathbf{g}(\mathbf{x}(s))+\mathbf{P}^{\mathbf{r}} \mathbf{H}(\mathbf{x}(s))\right] \mathbf{x}^{\prime}(s) .
$$

The tangent $\mathbf{x}^{\prime}(s)$ again is a contravariant vector. The first summand at the right hand side will be estimated to be zero because $\mathbf{P}^{\mathbf{r}}$ changes only slowly with $s$. The approximation concerns only the predictor step, not the exactness of the corrector. Thus we may assume that the older equation (3) again works well. It is an homogeneous system of equations and it is nontrivially solvable at regular curve points because we have $(n-1)$ equations and $n$ unknowns. So we may continue here as in section 3 .

RGFs are defined by equations (2) and (5). However, they are also solutions of the differential equation of Branin (7) using the adjoint matrix A. The property (6) holds. $\mathbf{H}$ is a two-fold covariant tensor. To fulfill equation (6) under any coordinate transformation: giving a "constant" matrix on the right-hand side, the matrix A has to be a two-fold contravariant tensor. Thus, in equation (7), the matrix A shifts the covariance of $\mathbf{g}$, and saves the contravariant character of $\mathbf{x}^{\prime}$. In contrast to the steepest descent $[6,42]$, no additional use of the metric is needed.

\section{The invariance of RGF: a second algorithm for a dyadic projector}

For the invariant definition of RGFs using the projector (4) in equation (5), we have to start with a covariant unit vector $\mathbf{r}=\mathbf{r}_{\text {cov }}$ for the search direction because we will compare it with the covariant gradient. Additionally, we have $\mathbf{r}^{\text {contra }}$ by (17). We use for the transpose $\mathbf{r}^{T}$ the contravariant form in the projector

$$
\mathbf{P}^{\mathbf{r}}:=\mathbf{I}-\mathbf{r}_{\operatorname{cov}} \mathbf{r}^{\text {contra } T} .
$$

It is $\mathbf{P}^{\mathbf{r}} \mathbf{r}_{\text {cov }}=\mathbf{0}$. The projector again has to be recalculated at new curve points because the metric changes from point to point. Because the metric usually changes slowly along an RP, the change of $\mathbf{r}^{\text {contra }}$ is also slowly. $\mathbf{P}^{\mathbf{r}}$ may be seen to be an invariant tensor because a coordinate transformation is possible in equation (23). The dyadic product makes that $\mathbf{P}^{\mathbf{r}}$ is a mixed tensor with one 
covariant and one contravariant index. $\mathbf{P}^{\mathbf{r}}$ is applicable to the covariant gradient in equation (5), as well as to the two-fold covariant Hessian in equation (3). It does not change the character of these arguments after application. Then an RGF calculation is invariant under coordinate transformation.

The dyadic matrix $\mathbf{r}_{\mathrm{cov}} \mathbf{r}^{\mathrm{contra} T}$ has rank 1 , and the projector rank $(n-1)$. RGFs are defined by equation (5). The condition defines a one-dimensional curve of points, $\mathbf{x}(s)$. The points in the gradient function are contravariant vectors. The procedure for the path following gives $\mathbf{x}(s)$. Equation (5) is automatically fulfilled in stationary points. The tangent for the predictor step is to grasp by equation (22). The tangent $\mathbf{x}^{\prime}(s)$ is a contravariant vector. Again, the first summand will be estimated to be zero because $\mathbf{r}^{\text {contra }}$ changes slowly with $s$. Thus we reduce equation (22) to the old equation (3). It is an homogeneous system of equations and it is non trivially solvable because $\mathbf{P}^{\mathbf{r}}$ has a rank deficit of one. The matrix $\left(\mathbf{P}^{\mathbf{r}} \mathbf{H}\right)$ is singular per definition, and a way to solve the system is the singular value decomposition (SVD) [43]. The SVD of any $(n \times n)$ matrix $\mathbf{S}$ is the decomposition

$$
\mathbf{S}=\mathbf{U}[\Sigma] \mathbf{V}^{T},
$$

where $\mathbf{U}$ and $\mathbf{V}$ are orthonormal matrices and $[\Sigma]$ is a diagonal matrix of the singular values $\sigma_{k k} \geqslant 0$ [43]. Only if $\mathbf{S}$ is a singular matrix, then a row with number $\mathrm{z}$ of $\mathbf{V}^{T}$ to the singular value $\sigma_{z z}=0$ gives the solution of the problem

$$
\mathbf{S t}=\mathbf{0} .
$$

We have to use $\mathbf{t}=\mathbf{v}_{z}$. It is $\mathbf{V}^{T} \mathbf{t}=\{0, \ldots, 1, \ldots, 0\}^{T}$, where the 1 is at place z. This single 1 meets the singular value $\sigma_{z z}=0$, and the zero equation (25) is fulfilled. In (24) the zero singular values may be sorted into the last rows and columns. Then the first columns of $\mathbf{U}$ and $\mathbf{V}$ are orthonormal bases of the column space of $\mathbf{S}$ with

$$
\mathbf{S v}_{k}=\sigma_{k k} \mathbf{u}_{k}, \quad \sigma_{k k}>0 .
$$

The last columns of $\mathbf{U}$ and $\mathbf{V}$ are the orthonormal bases of the null space of $\mathbf{S}^{T}$ and $\mathbf{S}$ [43].

In curvilinear coordinates, we have to modify the ansatz of SVD. The metric forms a Riemannian space of the configurations, and the matrix $\left(\mathbf{P}^{\mathbf{r}} \mathbf{H}\right)$ has to be observed in its tensor character. We assume that $\mathbf{H}$ is two-fold covariant, $\mathbf{P}^{\mathbf{r}}$ is a mixed two-tensor: one index covariant, one index contravariant. Then $\left(\mathbf{P}^{\mathbf{r}} \mathbf{H}\right)$ again is two-fold covariant: one contravariant index of $\mathbf{P}^{\mathbf{r}}$ and one covariant of $\mathbf{H}$ "cancel out" by the matrix multiplication. Equation (25) is the product of the two-fold covariant $\left(\mathbf{P}^{\mathbf{r}} \mathbf{H}\right)$ with the contravariant tangent: a covariant vector. But because it is a zero vector, its character does not matter 


$$
\sum_{j=1}^{n}(p h)_{k j} t^{j}=0_{k}
$$

The comparison with (25) shows that possible covariant vectors $\mathbf{v}_{i}$ of $\mathbf{V}$ of the SVD (24) cannot be equalized with the contravariant $\mathbf{t}$ vector. The remedy is a more tricky use of the SVD in a generalized metric version [44]. We decompose the inverse metric matrix $\left(\mathrm{g}^{l m}\right)$ by Cholesky decomposition into a product of a lower and an upper triangular matrix.

$$
\left(\mathrm{g}^{l m}\right)=\mathbf{L L}^{T}=\left(\begin{array}{ccc}
L^{11} & . . & 0 \\
. . & . . & . . \\
L^{n 1} & . . & L^{n n}
\end{array}\right)\left(\begin{array}{ccc}
L^{11} & . . & L^{1 n} \\
. . & . . & . . \\
0 & . \cdot & L^{n n}
\end{array}\right),
$$

which means for the elements

$$
\mathrm{g}^{l m}=\sum_{k=1}^{n} L^{l k} L^{k m}=\sum_{k=1}^{n} L^{l k} L^{m k}
$$

The calculation of $\mathbf{L}$ starts with $L^{11}=\sqrt{\mathrm{g}^{11}}$ and obtains by iteration the further $L^{l m}$.

Matrix $\mathbf{L}$ is sometimes also described by $\left(\mathrm{g}^{l m}\right)^{1 / 2}$. We use the representation

$$
\left(\mathbf{P}^{\mathbf{r}} \mathbf{H}\right)=\mathbf{L}^{-T}\left(\mathbf{L}^{T}\left(\mathbf{P}^{\mathbf{r}} \mathbf{H}\right) \mathbf{L}\right) \mathbf{L}^{-1}
$$

and we put the internal part $\left(\mathbf{L}^{T} \mathbf{P}^{r} \mathbf{H} \mathbf{L}\right)$ into the usual SVD. It gets like equation (24)

$$
\left(\mathbf{L}^{T} \mathbf{P}^{\mathbf{r}} \mathbf{H L}\right)=\mathbf{U}[\Sigma] \mathbf{V}^{T}
$$

and in combination with (30) we obtain the decomposition

$$
\left(\mathbf{P}^{\mathbf{r}} \mathbf{H}\right)=\left(\mathbf{L}^{-T} \mathbf{U}\right)[\Sigma]\left(\mathbf{V}^{T} \mathbf{L}^{-1}\right)
$$

Setting

$$
\mathbf{L}^{-T} \mathbf{U}=\mathbf{U}_{\text {gen }} \quad \text { and } \quad \mathbf{L}^{-T} \mathbf{V}=\mathbf{V}_{\text {gen }}
$$

where the subscript "gen" means generalized for internal metric, gives the generalized SVD

$$
\left(\mathbf{P}^{\mathrm{r}} \mathbf{H}\right)=\mathbf{U}_{\text {gen }}[\Sigma] \mathbf{V}_{\text {gen }}^{T}
$$

The new decomposition matrices $\mathbf{U}_{\text {gen }}$ and $\mathbf{V}_{\text {gen }}$ fulfill an orthogonality condition, but within the correct metric part. It is with (28) and (32)

$$
\mathbf{U}_{\text {gen }}^{T}\left(\mathrm{~g}^{k l}\right) \mathbf{U}_{\text {gen }}=\left(\mathbf{U}^{T} \mathbf{L}^{-1}\right)\left(\mathbf{L L}^{T}\right)\left(\mathbf{L}^{-T} \mathbf{U}\right)=\mathbf{I}
$$


and it is analogously

$$
\mathbf{V}_{\text {gen }}^{T}\left(\mathrm{~g}^{k l}\right) \mathbf{V}_{\text {gen }}=\mathbf{I}
$$

$\mathbf{U}_{\text {gen }}$ and $\mathbf{V}_{\text {gen }}$ are orthonormal matrices with column vectors of covariant character and measure. Coming back to equation (27), we have to choose the tangent to be the contravariant version of the covariant column vector $\mathbf{v}_{\mathbf{z}}$ of $\mathbf{V}_{\text {gen }}$

$$
t^{k}=\sum_{l} \mathrm{~g}^{k l}\left(\mathbf{v}_{\mathbf{z}}\right)_{l} .
$$

We can multiply equation (34) by this t from the right hand side and get

$$
\left(\mathbf{P}^{\mathbf{r}} \mathbf{H}\right) \mathbf{t}=\mathbf{U}_{\mathrm{gen}}[\Sigma] \mathbf{V}_{\mathrm{gen}}^{T} \mathbf{t}=\mathbf{U}_{\mathrm{gen}}[\Sigma]\left(\begin{array}{c}
0 \\
\cdot \\
\cdot \\
1 \\
\cdot \\
\cdot \\
0
\end{array}\right)=\mathbf{0}
$$

where the 1 is at the $z$-th place. The tangent $\mathbf{t}$ is a contravariant vector and it is used as predictor direction for the predictor step. It is a contravariant basis vector of the null space of $\left(\mathbf{P}_{r} H\right)$.

After doing the predictor step, $\mathbf{x}_{m+1}=\mathbf{x}_{m}+l \mathbf{t}_{m}$, we may be out of the searched curve. It means the reduced gradient is not the zero vector in the metric of curvilinear coordinates

$$
\mathbf{P r}^{\mathbf{r}} \mathbf{g}\left(\mathbf{x}_{m+1}\right) \neq \mathbf{0}
$$

It is a covariant vector

$$
\left(\mathbf{P}^{\mathbf{r}} \mathbf{g}\right)=\left(\sum_{j} p_{k}^{j} g_{j}\right)=\left(\gamma_{k}\right) .
$$

To find the step back to the RGF curve we try to solve a Newton-Raphson step $\Delta \mathbf{x}$ by

$$
\left(\mathbf{P}^{\mathbf{r}} \mathbf{H}\right) \Delta \mathbf{x}=-\mathbf{P}^{\mathbf{r}} \mathbf{g} .
$$

However, the matrix $\left(\mathbf{P}^{r} H\right)$ is singular per definition, and there is only a pseudo-inverse which we can use. With the generalized SVD we construct the solution. From (35) we know that $\mathbf{U}_{\text {gen }}^{T}\left(\mathrm{~g}^{k l}\right)$ is the inverse of $\mathbf{U}_{\text {gen }}$ in the Riemannian metric, and from (36) we know that $\left(\mathrm{g}^{k l}\right) \mathbf{V}_{\text {gen }}$ is the inverse of $\mathbf{V}_{\text {gen }}^{T}$ in the Riemannian metric. The pseudo-inverse of $[\Sigma]$ with at least one zero 
singular value is that diagonal matrix where only the non-zero elements are inverted:

$$
[\Sigma]^{+}=\left[1 / \sigma_{k k}\right] \text { for } k k \neq z z
$$

but again the $z$-th row is a zero row. Rewriting equation (41) now is

$$
\left(\mathbf{U}_{\text {gen }}[\Sigma] \mathbf{V}_{\text {gen }}^{T}\right) \Delta \mathbf{x}=-\left(\mathbf{P}^{\mathbf{r}} \mathbf{g}\right)
$$

Successively, we apply from the left-hand side: first $\mathbf{U}_{\text {gen }}^{T}\left(\mathrm{~g}^{k l}\right)$, a matrix of contravariant vectors, thereafter $[\Sigma]^{+}$, and at last $\left(\mathrm{g}^{k l}\right) \mathbf{V}_{\text {gen }}$, again a matrix of contravariant vectors. We refer to equation (44) as the solution for the corrector step

$$
\Delta \mathbf{x}=-\left[\mathbf{U}_{\text {gen }}^{T} \mathrm{~g}^{k l}\right][\Sigma]^{+}\left[\mathrm{g}^{k l} \mathbf{V}_{\text {gen }}\right]\left(\mathbf{P}^{\mathbf{r}} \mathbf{g}\right) .
$$

The step results as a contravariant vector, as it is expected for the corrector in a coordinate space. Equation (44) is applied until convergence.

\section{Example}

It is natural to ask how the intrinsic metric compares to the previous method in terms of performance. An objective comparison does not seem straight-forward since there are different possibilities of internal coordinates. We use the HCN $6-31 G^{*}$ potential surface as our illustrative vehicle. The isomerization pathway serves as a numeric example. Figure 1 shows calculated RGF pathways in the HCN configuration space in internal coordinates. The distances of $\mathrm{CH}$ and $\mathrm{CN}$ are given in $\AA$, and $\alpha$ is the angle between the bonds. Curve $\mathbf{a}$ is the Newton trajectory calculated with the help of projector (15) in the correct metric version. Curve b uses the RGF with the dyadic projector (23) in the metric version, however, $\mathbf{c}$ uses the RGF with projector (2) in the "Cartesian" metric version using a unit matrix instead of $\left(\mathrm{g}^{i j}\right)$. The search direction $\mathbf{r}$ of equation (1) is the gradient at a point close to the $\mathrm{HNC}$ minimum distorted by $1^{\circ}$ in the bending direction $\alpha$. The search is from bottom to top in figure 1. It leads from HNC minimum up in energy to the SP, but then downhill in energy to the next minimum, the HCN valley. (The calculation is not terminated at SP.) The CN distance is nearly constant along the pathway. For the step length of the predictor steps we use a large $l=0.5$ in internal units, and we put a small $\epsilon=0.0005$ for the threshold of the corrector. (It is less than the proposal for RGF [9] being $\epsilon \approx l / 100$, however, compare the still better ratio $\approx l / 1$ given in [22]). Figure 1 shows the expected results: Curves $\mathbf{a}$ and $\mathbf{b}$ do nearly coincide, where $\mathbf{c}$ goes besides. Note that case $\mathbf{c}$ misses the SP and the HCN minimum. Step length and corrector threshold are too large for this coarse approximation. Because the internal coordinates are two distances and one angle, their measure should not be reduced to the Cartesian norm! The progress of the pathway is mainly the 


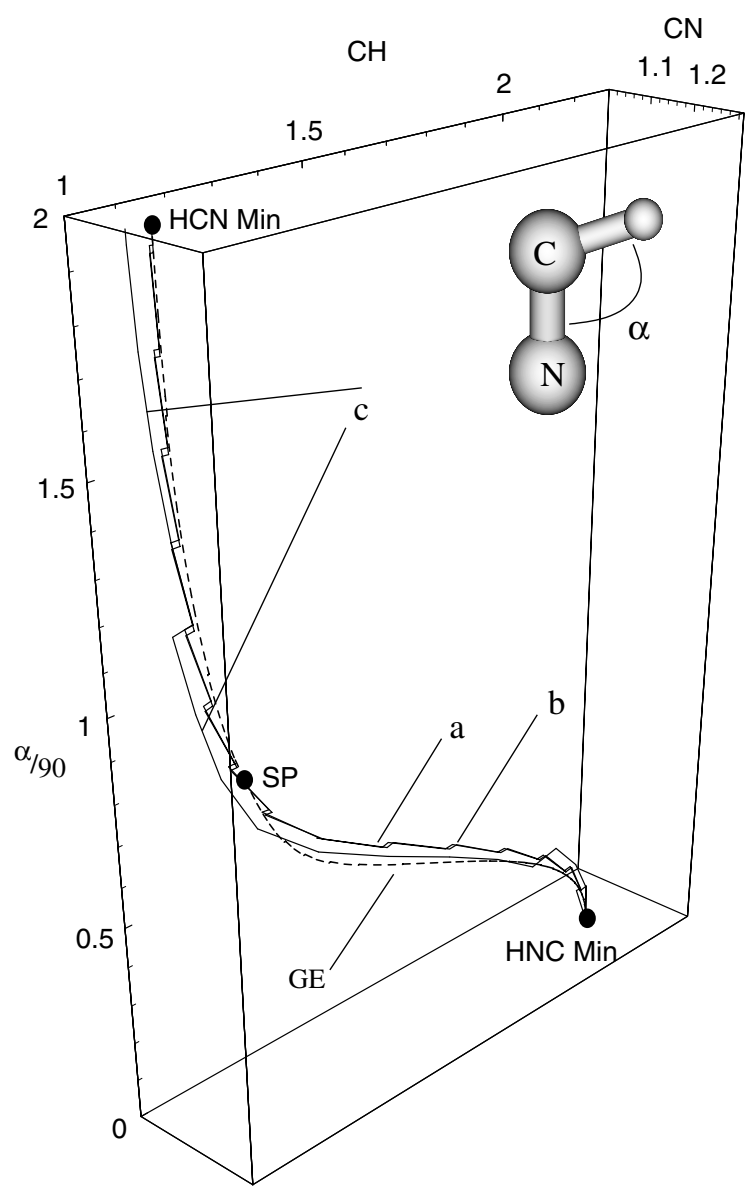

Figure 1. Clumsy approximations of RGFs on the PES of HCN isomerization given in the 3-D configuration space of distances $\mathrm{CH}$ and $\mathrm{CN}$ (in $\AA$ ), and angle $\alpha$ (scaled by $1 / 90$ ). Shown are predictor and corrector steps. a and $\mathbf{b}$ use the projectors (15) and (23), respectively, in the correct internal metric. $\mathbf{c}$ uses the projector (2) in a "Cartesian" metric version. The dashes are the gradient extremal for comparison giving the valley ground line.

angle, and if the norms are calculated with the exact Riemannian metric we get the more exact path following. The gradient extremal is given for orientation in the HCN mountains, for the valley ground line of the isomerization. Usually, RGFs are not on the gradient extremal [42].

\section{Discussion}

An RGF line may be understood to be a line where the uphill driving force has always the same direction, the force which has to overpower the gradient. 
RGF is a pre-dynamical allegory for a chemical reaction path like the IRC, the steepest descent from saddle point.

We have extended the algorithms for RGF to the use of internal coordinates. The Newton trajectories can follow loose RP definitions, as exactly as one needs it, to find a corresponding SP by going uphill, or a minimum by going downhill. RGF curves are definably independent of the coordinate system. To circumvent the usual way of following a curve by a projection into an $(n-1)$ dimensional subspace [19], we propose and apply a generalized singular value decomposition (SVD) in the full $n$-dimensional curvilinear space. It is a new mathematical tool for the path following along a given definition of a curve.

Next to the problem of defining a suitable curve for the reaction path of chemistry is the possibility of RP branching. The corresponding points are bifurcation points. The branching of Newton trajectories is connected with the valley-ridge inflection points of the PES. The method of following a reduced gradient as well as the Branin method have succeeded in computing symmetric VRI points. (Un-symmetric VRIs can be found by following the valley extremals [26].)

\section{Acknowledgments}

The work was made possible through the financial support of the Deutsche Forschungsgemeinschaft. The author thanks $M$. Hirsch for stimulating discussions.

\section{References}

[1] K. Laidler, Theory of Reaction Rates (McGraw-Hill, New York, 1969).

[2] W. Miller, N.C. Handy and J.E. Adams, J. Chem. Phys. 72 (1980) 99.

[3] P.G. Mezey, Potential Energy Hypersurfaces (Elsevier, Amsterdam, 1987).

[4] D. Heidrich, W. Kliesch and W. Quapp, Properties of Chemically Interesting Potential Energy Surfaces (Springer, Berlin, 1991).

[5] D. Heidrich, The Reaction Path in Chemistry: Current Approaches and Perspectives (Kluwer, Dordrecht, 1995).

[6] W. Quapp and D. Heidrich, Theor. Chim. Acta 66 (1984) 245.

[7] I.H. Williams and G.M. Maggiora, J. Mol. Struct. (Theochem) 89 (1982) 365.

[8] W. Quapp, M. Hirsch, O. Imig and D. Heidrich, J. Comput. Chem. 19 (1998) 1087.

[9] W. Quapp, M. Hirsch and D. Heidrich, Theor. Chem. Acc. 100 (1998) 285.

[10] M. Hirsch, W. Quapp and D. Heidrich, Phys. Chem. Chem. Phys. 1 (1999) 5291.

[11] J.M. Anglada, E. Besalu, J.M. Bofill and R. Crehuet, J. Comput. Chem. 22 (2001) 387.

[12] J.M. Bofill and J.M. Anglada, Theor. Chem. Acc. 105 (2001) 463.

[13] R. Crehuet, J.M. Bofill and J.M. Anglada, Theor. Chem. Acc. 107 (2002) 130.

[14] W. Quapp, E-mail: quapp@rz.uni-leipzig.de Web: www.mathematik.uni-leipzig.de/MI/quapp/mrgf

[15] K. Müller, Angew. Chem. 92 (1980) 1.

[16] K. Müller and L.D. Brown, Theor. Chim. Acta 53 (1979) 75.

[17] J. Cioslowski, A.P. Scott and L. Radon, Mol. Phys. 91 (1997) 413. 
[18] A. Kiełbasiński and H. Schwetlick, Numerische Lineare Algebra (Deutscher Verl. Wiss., Berlin, 1988).

[19] E.L. Allgower and K. Georg, Numerical Continuation Methods - An Introduction (Springer, Berlin, 1990).

[20] W. Quapp, J. Comput. Chem. 22 (2001) 537.

[21] D. Lauvergnat, A. Nauts, Y. Justum and X. Chapuisat, J. Chem. Phys. 114 (2001) 6592.

[22] M. Hirsch and W. Quapp, J. Comput. Chem. 23 (2002) 887.

[23] K. Schiele and R. Hemmecke, Z. Angew. Math. Mech. 81 (2001) 291.

[24] M. Dallos, H. Lischka, E. Ventura do Monte, M. Hirsch and W. Quapp, J. Comput. Chem. 23 (2002) 576.

[25] W. Quapp and D. Heidrich, J. Mol. Struct. (Theochem) 585 (2002) 105.

[26] W. Quapp, M. Hirsch and D. Heidrich, Theor. Chem. Acc. 112 (2004) 40; W. Quapp, J. Mol. Struct. 695-696 (2004) 95.

[27] H. Metiu, J. Ross, R. Silbey and T.F. George, J. Chem. Phys. 61 (1974) 3200.

[28] P. Valtazanos and K. Ruedenberg, Theor. Chim. Acta 69 (1986) 281.

[29] F.H. Branin, IBM J. Res. Dev. 16 (1972) 504.

[30] H.T. Jongen, P. Jonker and F. Twilt, Nonlinear Optimization in Finite Dimensions - Morse Theory, Chebychev Approximation, Transversality, Flows, Parametric Aspects (Kluwer, Dordrecht, 2000).

[31] I. Diener and R. Schaback, J. Optimiz. Theory Appl. 67 (1990) 87; I. Diener, Globale Aspekte des kontinuierlichen Newton-Verfahrens (Habilitation, Göttingen, 1991).

[32] W. Quapp and V. Melnikov, Phys. Chem. Chem. Phys. 3 (2001) 2735.

[33] D.J. Wales, J. Chem. Phys. 113 (2000) 3926.

[34] W. Quapp, J. Chem. Phys. 114 (2001) 609.

[35] W. Quapp, J. Chem. Soc. Faraday Trans. 90 (1994) 1607.

[36] W. Quapp, in: The Reaction Path in Chemistry: Current Approaches and Perspectives, ed. D. Heidrich (Kluwer, Dordrecht, 1995) pp. 95-107.

[37] E.B. Wilson, J.C. Decius and P.C. Cross, Molecular Vibrations (McGraw-Hill Comp., New York, 1955).

[38] A. Tachibana and K. Fukui, Theor. Chim. Acta 49 (1978) 321.

[39] V. Bakken and T. Helgaker, J. Chem. Phys. 117 (2002) 9160.

[40] H. Dachsel and W. Quapp, J. Math. Chem. 6 (1991) 77.

[41] B.P. Winnewisser and J.K.G. Watson, J. Mol. Spectrosc. 205 (2001) 227.

[42] W. Quapp, J. Theor. Comp. Chem. 2 (2003) 385.

[43] W.H. Press, B.P. Flannery, S.A. Teukolsky and W.T. Vetterling, Numerical Recipes (Cambridge University Press, Cambridge, 1989).

[44] D.G. Leibovici, http://www.fmrib.ox.ac.uk/ didier/ 